\title{
Tocotrienol Rich Fraction from Palm Oil Reduces Plasma and Erythrocyte Membrane Lipid Alteration in Diabetic Rats: Tocotrienols Reduce Dyslipidemia in Plasma and Erythrocytes
}

\author{
Nasar Alwahaibi ${ }^{1, *}$, Siti Balkis Budin², Zariyantey Abdul Hamid ${ }^{2}$, Jamaludin Mohamed², \\ Jalifah Latip ${ }^{3}$, Norzamzurina Binti Ismail ${ }^{4}$, Alefee Bin Ayatillah ${ }^{4}$, Fatmah Ali Matough ${ }^{2}$ \\ ${ }^{1}$ Department of Allied Health Sciences, College of Medicine and Health Sciences, Sultan Qaboos University, Muscat, Oman \\ ${ }^{2}$ Program of Biomedical Sciences, School of Diagnostic and Applied Health Sciences, Faculty of Health Sciences, Universiti Kebangsaan Malaysia, \\ Bangi, Malaysia \\ ${ }^{3}$ School of Chemical Science and Food Technology, Faculty of Science and Technology, Universiti Kebangsaan Malaysia, Bangi, Malaysia \\ ${ }^{4}$ Center for Research and Instrumentation Management, Universiti Kebangsaan Malaysia, Bangi, Malaysia \\ *Corresponding author. E-mail: nasar@squ.edu.om
}

Received date: Dec 25, 2018; Revised date: Apr 8, 2019; Accepted date: Apr 8, 2019

\section{Abstract}

$\mathrm{B}$ ACKGROUND: Tocotrienol-rich fraction (TRF) from palm oil has been shown to exhibit potent antioxidative properties in various pathological conditions. This study aimed to determine the antioxidative properties of TRF on the plasma and erythrocyte membrane lipids composition in streptozotocin-induced diabetic rats.

METHODS: Male Sprague-Dawley rats $(\mathrm{n}=40)$ were divided randomly into four groups of 10 , which were a normal group, a normal group with TRF, a diabetic group, and a diabetic group with TRF. Following four weeks of treatment, lipids compositions in the plasma and erythrocyte membrane were investigated.

RESULTS: TRF significantly reduced the plasma total cholesterol, to that in the diabetic group. In addition, supplementation of TRF was able to reduce the very low density lipoprotein cholesterol (VLDL-C), triglycerides levels in treated diabetic rat although no significant different was observed when compared with untreated diabetic rats alone. Furthermore, TRF supplementation showed increased the levels of phosphatidylinositol, phosphatidylserine, and total phospholipid levels in diabetic rats. However, the linoleic acid (18:2) levels were reduced in diabetic rats compared to the control group, and the eicosadienoic acid (20:2) levels were significantly increased by TRF supplementation in diabetic rats.

CONCLUSION: Supplementation with $200 \mathrm{mg} / \mathrm{kg}$ TRF improves dyslipidemia and that TRF, therefore, might have protective effects against oxidative damage of phospholipids. This study also showed the antioxidative properties of TRF on the erythrocyte membrane lipids composition, in particular, its protective effects against peroxidation of unsaturated fatty acids.

KEYWORDS: antioxidative property, cholesterol, fatty acids, phospholipids, tocotrienol-rich fraction

Indones Biomed J. 2019; 11(3): 247-56

\section{Introduction}

Various studies showed that antioxidant vitamins and supplements can reduce the markers indicative of oxidant stress and lipid peroxidation in diabetic subjects and animals.(1) The nutritional role of vitamin $\mathrm{E}$ is clearly that of a biological antioxidant. Vitamin $\mathrm{E}$ is believed to have basic functional importance in the maintenance of membrane integrity in almost all cells of the body. It reduces free radicals and therefore protects against the deleterious reactions of highly reactive oxidizing species.(2) 
Palm oil, annatto, rice bran oil, oats, hazelnuts and maize are rich natural sources of vitamin E, mainly tocotrienol.(1) A better understanding of the biological significant of tocotrienols should contribute not only to the knowledge of discovering the health-giving properties of tocotrienols, but also towards more effective potential therapeutic agent in the prevention of several pathological condition and maintenance of human health. A number of health-related biological properties of tocotrienols have been identified, including its anticancer, anticholesterolemic, antihypertensive, antioxidant, immunomodulatory and neuroprotective properties.(3) In addition to its activities, tocotrienol-rich fraction (TRF), extracted from palm oil, inhibits cholesterol synthesis by suppressing 3-hydroxy3-methylglutaryl coenzyme A reductase in various cells. (4) Currently TRF addressed as anti-apoptotic and antiinflammatory agents.(5)

Recent review showed that tocotrienols have more antioxidant and free radical scavenging properties than tocopherols.(6) Thus, this study aimed to determine the antioxidant properties protective effects of TRF on plasma and erythrocyte membrane lipids composition of streptozotocin (STZ)-induced diabetic rats.

\section{Methods}

\section{Animals and Ethics Statement}

Forty male Sprague-Dawley rats, aged 8-10 weeks and weighed 200-250g, were provided by Laboratory Animal Resources Unit, Faculty of Medicine, Universiti Kebangsaan Malaysia. They were housed in plastic cages (two rats per cage) with wood chips for bedding. and were fed standard diet (mouse pellet 702 P, Gold Coin Sdn. Bhd., Selangor, Malaysia) and tap water ad libitum. The animals were acclimatized to standard laboratory conditions (temperature $25^{\circ} \mathrm{C}$, with light-darkness cycles of 12 hour) for one week before the commencement of the experiments. The recommendation of University Kebangsaan Malaysia Animal Ethics Committee (UKMAEC) for the care and use of the animals were followed throughout the study and the approval code is as follow (UKMAEC No.: FSKB

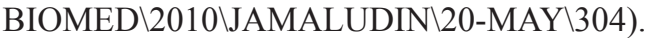

\section{TRF Supplementation in Rats}

Rats were randomly divided into four groups of 10 rats, as follows: normal rats served as control group (Group 1), normal rats supplemented with TRF (Group 2), non-treated diabetic group (Group 3), and diabetic rats supplemented with TRF (Group 4). Induction of diabetes was achieved following an overnight fasting by a single intravenous injection (via tail vein) of freshly prepared STZ (streptozotocin) (Sigma, St, Louis, USA) at a dose of 45 $\mathrm{mg} / \mathrm{kg}$ body weight (BW) which was freshly dissolved in normal saline. Three days later, blood collected via tail vein and glucose concentration was measured by a strip-operated blood glucose sensor (Companion 2, Medisense Ltd., and Birmingham, UK). Several studies reported that induction of experimental diabetes in the rat using STZ is very convenient and simple to use.(7,8) STZ injection leads to the degeneration of the Langerhans islets beta cells and lead to the reduction of insulin secretion in STZ induced diabetic rats. This mechanism cause increased in plasma glucose levels, which lead to diabetes condition. $(9,10)$ Clinically, symptoms of diabetes are clearly seen in rats within 2-4 days following single intravenous or intraperitoneal injection of $45 \mathrm{mg} / \mathrm{kg} \mathrm{STZ}$. The results of fasting blood glucose (FBG) presented in this study indicated that STZinduced diabetes was successfully achieved as evidenced by significantly high level of blood glucose in diabetic rats. This could be due to the inhibition of insulin secretion resulting from injection of STZ. Our study showed higher levels of glycemic status and food and fluid intake as well as decreased body weight, which had been reported in the past. Rats with blood glucose levels $>7.0 \mathrm{mmol} / \mathrm{L}$ were included in the study. $(11,12)$ The dose of TRF, which was obtained from SIME DARBY Malaysia, was administered orally at a dose of $200 \mathrm{mg} / \mathrm{kg}$ body weight /day $(7,9)$ throughout the feeding period of four weeks and the supplementation begun on the same day. Following four weeks of TRF supplementation, the rats were fasted overnight, and blood samples were collected by cardiac puncture under diethyl ether-induced deep anesthesia.

\section{Preparation of Erythrocytes Membranes and Extraction of Lipids}

Erythrocytes were centrifuged at $4000 \mathrm{rpm}$ for $15 \mathrm{~min}$ at $4^{\circ} \mathrm{C}$. The plasma was transferred into new $1.5 \mathrm{~mL}$ Eppendorf tubes and frozen until further use at $-80^{\circ} \mathrm{C}$. Extraction of erythrocytes membranes was performed according to the method described by Dodge and Phillips.(13) The erythrocytes-containing pellet was washed twice with cold $\left(4^{\circ} \mathrm{C}\right) 9 \%$ normal saline $(\mathrm{pH} 7.4)(5 / 1 \mathrm{v} / \mathrm{v})$. For the third wash, 5\% Trolox (97\% 6-hydroxy 5, 7, 8-tetramethylchroman2 -carboxylic acid) in normal saline was used in order to prevent oxidation of the membrane fatty acids. Lipids were extracted from the erythrocytes membranes using the method described previously.(14) 
Determination of The Lipids Composition in The Plasma High-density Lipoprotein (HDL), Low-density Lipoprotein (LDL), VLDL, and Total Cholesterol (TC) Plasma TC, HDL-C, and LDL, VLDL-C were quantified using a commercial enzymatic assay kit (Cat No. K613-100, Biovision Inc, Milpitas, USA). The readings for samples and the standard were determined using a fluorescence plate reader equipped with an excitation wavelength/emission wavelength $(\mathrm{Ex} / \mathrm{Em})$ of 538/587 nm (SkanIt Software, Thermo Fisher Scientific Inc, Waltham, USA).

\section{Measurement of Plasma Triglyceride (TG)}

TG levels were measured using an enzymatic-based hydrolysis method (Cat No. T532-480, Teco diagnostics, Anaheim, USA). TG are hydrolyzed by a lipase, into glycerol. The glycerol concentration is then determined using an enzymatic assay leading to the formation of a quinoneimine dye. Amount of dye produced was determined using a spectrophotometer $(520 \mathrm{~nm})$ and is directly proportional to the concentration of TG in the samples.

\section{Measurement of Plasma Total Lipid}

The total amount of lipids in the plasma was quantified using a sulfo-phospho-vanillin colorimetric method (Cat No. T526-480, Teco diagnostics). The amount of the purple complex formed was determined using a spectrophotometer at $530 \mathrm{~nm}$.

Determination of Erythrocyte Membrane Cholesterol and Phospholipids Using Liquid Chromatography-tandem Mass Spectrometry (LC-MS/MS) Instrumentation

We used a Thermo Scientific C18 SIRI Dionex Ultimate 3000 liquid chromatography system (Shimadzu CL 09115047, Shimadzu, Kyoto, Japan), directly coupled to the mass spectrometer system MS (Bruker/microTof Q, Bruker Daltonics, Billerica, USA), equipped with an electro spray ionization (ESI) source. We used the positive mode on a 150 x $2 \mathrm{~mm}$ Phenomenex C18 reversed-phase column $(2.0 \mathrm{~mm}$ x $4.6 \mathrm{~mm}$ ) of $5-\mu \mathrm{m}$ particle size. LC-MS was performed using an integrated pre column (SepServ, Berlin, Germany). The column was maintained at $60^{\circ} \mathrm{C}$ and we used a mobile phase flow-rate of $0.3 \mathrm{~mL} / \mathrm{min}$. MS data were acquired over a scan range of $50-1500 \mathrm{~m} / \mathrm{z}$. The other parameters used are as follows: Set Capillary $4000 \mathrm{~V}$, End Plate Offset -500 V, Set Collision Cell RF 250.0 Vpp.

\section{Erythrocyte Membrane Phospholipids Analysis}

The separation of phospholipids was performed using the method conducted by Alefee.(15) The mobile phase was composed of solvent A ( $0.1 \%$ formic acid in water) and solvent $\mathrm{B}$ (methanol, $\mathrm{MeOH}$ ). Both were freshly prepared prior to analysis. The solvents and the flow rates used were as shown in Table 1. The lipids extracted were filtered using a high performance liquid chromatography (HPLC) $(45-\mu \mathrm{m})$ filter prior to injection into the LC-MS system. Separation was carried out using a flow rate of $0.3 \mathrm{~mL} / \mathrm{min}$ and an evaporative light scattering detector was used. Data were analyzed using the Bruker Daltonics software (version 3.4) to quantify the average of phospholipids levels in $\mu \mathrm{g} /$ $\mathrm{mg}$ of proteins.

Table 1. Solvent gradient and flow rates used for separation of phospholipids by LC-MS.

\begin{tabular}{cccc}
\hline $\begin{array}{c}\text { Time } \\
(\mathbf{m i n})\end{array}$ & $\begin{array}{c}\text { Solvent A } \\
(\mathbf{\%})\end{array}$ & $\begin{array}{c}\text { Solvent B } \\
\mathbf{( \% )}\end{array}$ & $\begin{array}{c}\text { Flow-rate } \\
(\mathbf{m L} / \mathbf{m i n})\end{array}$ \\
\hline 0 & 90 & 10 & 0.3 \\
5 & 90 & 10 & 0.3 \\
15 & 0 & 100 & 0.3 \\
25 & 0 & 100 & 0.3 \\
26 & 90 & 10 & 0.3 \\
30 & 90 & 10 & 0.3 \\
\hline
\end{tabular}

\section{Erythrocyte Membrane Cholesterol Analysis}

The lipid extracts were resuspended in $1 \mathrm{~mL}$ of ethanol and homogenized using a vortex for $15 \mathrm{sec}$. Standard concentrations ranging from $0,10,20,40,80$ and $100 \mu \mathrm{g}$ of lipids were (Table 2). After resuspension, the samples were filtered using an HPLC $(45-\mu \mathrm{m})$ filter and injected into the LC-MS system. We followed the same procedure as described for erythrocyte membrane phospholipids analysis. The cholesterol concentration was calculated as $\mu \mathrm{g} / \mathrm{mg}$ protein. The retention time for cholesterol was 15 $\min$.

Table 2. Cholesterol standard concentrations.

\begin{tabular}{ccc}
\hline $\begin{array}{c}\text { Standard Solution } \\
\text { Concentration } \\
(\boldsymbol{\mu} \mathbf{g} / \mathbf{m L})\end{array}$ & $\begin{array}{c}\text { Volume of Stock } \\
\text { Cholesterol } \\
(\mathbf{m L})\end{array}$ & $\begin{array}{c}\text { Volume of } \\
\text { Ethanol } \\
(\mathbf{m L})\end{array}$ \\
\hline 0 & 0 & 2 \\
10 & 0.2 & 1.8 \\
20 & 0.4 & 1.6 \\
40 & 0.8 & 1.2 \\
80 & 1.6 & 0.4 \\
100 & 2 & 0 \\
\hline
\end{tabular}


Erythrocyte Membrane Fatty Acid Composition Analysis: Preparation of Fatty Acid Methyl Esters from Lipids with Boron Fluoride-methanol

Prior to gas chromatography (GC), methylation of fatty acids was carried out using a boron trifluoride-methanol reagent in accordance with the method described by Morrison and Smith.(6) Briefly, the extracted lipids were evaporated to dryness under nitrogen in a centrifuge tube. Then, $1 \mathrm{~mL}$ of the boron fluoride-methanol reagent was added under nitrogen gas, and the tubes were closed with the screw cap. The tubes were then heated at $100^{\circ} \mathrm{C}$ in a boiling water bath for $2 \mathrm{~min}$. The esters were extracted using 2 volumes of pentane and 1 volume of water. After homogenization, the samples were centrifuged until both layers were well separated and clear (this can give up to $97-99 \%$ extraction of the esters). The upper layer, containing the pentane and extracted lipids, was transferred into a new test tube, and the total fatty acids composition was determined by GC-MS system.

\section{GC-MS Instrumentation}

For the gas chromatography-mass spectrometry (GC/MS) analysis, we used an Agilent 7890A Gas Chromatograph (GC) directly coupled to the mass spectrometer system (MS). The parameters were as follows: Agilent 5975C inert inert mass selective detector (MSD) with triple-axis detector, column model SP-2380, length $30 \mathrm{~m}$, diameter $0.25 \mathrm{~mm}$, stationary phase, stabilized, poly ( $90 \%$ biscyanopropyl $/ 10 \%$ cyanopropylphenyl siloxane, film thickness $0.25 \mu \mathrm{m}$, Corporation Supelco Analytical. The fatty acids composition was determined according to previously described method. (17) After injecting $1 \mu \mathrm{L}$ of each FAME extract into the GC system. The oven temperature was programmed to go up from $70^{\circ}$ to $160^{\circ} \mathrm{C}$ at $25^{\circ} \mathrm{C} / \mathrm{min}$ and then from $160^{\circ} \mathrm{C}$ to $250^{\circ} \mathrm{C}$ at $3^{\circ} \mathrm{C} / \mathrm{min}$. The temperature was then maintained at $250^{\circ} \mathrm{C}$ for $20 \mathrm{~min}$. The helium gas flow rate used was 1.5 $2.0 \mathrm{~mL} / \mathrm{min}$, the flame ionization temperature $300^{\circ} \mathrm{C}$ and the temperature of the injector was $240^{\circ} \mathrm{C}$.

\section{Identification of Peaks}

The MSD Chemstation was used to analyze the GC chromatograms. A library search was carried out using the NIST/EPA/NIH Version 2.0, and the results were combined in a single peak table.

\section{Statistical Analysis}

The data were analyzed using SPSS version 16 (SPSS Inc., Chicago, USA). Shapiro-Wilk test was used to check the normality of the variable. Accordingly, ANOVA were used to analyze data follow normal behaviour of distribution pattern, followed by post hoc LSD multiple comparison test were used to estimate the significance different between groups. While, Kruskal-Wallis-one way ANOVA test were used to analyze data follow non-normal behaviour of distribution pattern. The difference between groups was considered significant when $p<0.05$.

\section{Results}

\section{Body Weight, Food and Water Consumption}

Throughout four weeks of study, the food and water intake were markedly increased in diabetic groups (diabetic and diabetic with TRF) when compared to those normal groups. The average of food intakes for all diabetic groups were 642.1-902 g per week while normal groups showed an average of 230.5-324.9 g per week. Water intakes for all diabetic groups had average of 350-600 mL per week, while normal groups showed an average of 160-290 mL per week. The consumptions of food and water were calculated per cage so that statistical result would not be influenced. Body weight for all experimental groups was recorded throughout the period of four week. Initially, all experimental groups had similar body weights, at the end of the study period both diabetic groups (untreated diabetic and diabetic with TRF) showed significantly loss of body weight when compared to the normal groups (Figure 1).

\section{Effects of TRF Supplementation on Plasma Lipoprotein Profiles (HDL-C, LDL-C, VLDL-C)}

Untreated diabetic group showed significantly lower plasma HDL-C concentration when compared to the control group $(p=0.03)$. However, TRF-treated diabetic rats showed significantly higher levels of HDL-C when compared to the untreated diabetic group $(p=0.002)$. Plasma LDL-C levels were higher in diabetic rats but none significantly different when compared to the control groups, TRF-treated diabetic rats showed a significantly lower level of LDL-C when compared to the untreated diabetic rats $(p=0.0001)$. The levels of VLDL-C were significantly higher in both treated and non-treated diabetic groups compared to the control group ( $p=0.0001$ for both). However, TRF treatment was able to reduce the VLDL-C levels in treated diabetic rat although no significant different was observed when compared with untreated diabetic rats alone (Figure 2A, 2B and $2 \mathrm{C}$ ). 

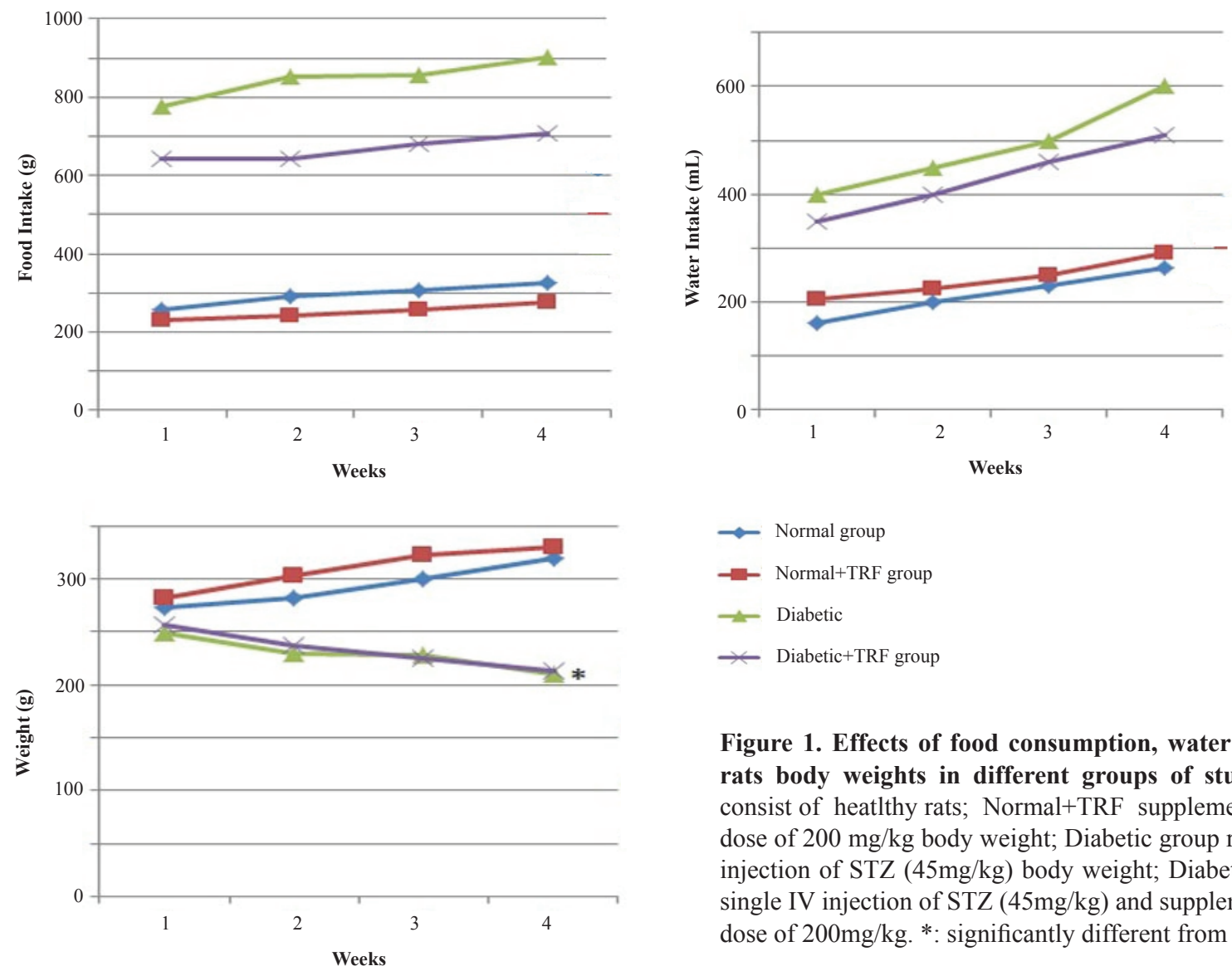

Figure 1. Effects of food consumption, water consumption and rats body weights in different groups of study. Normal group consist of heatlthy rats; Normal+TRF supplemented with TRF at dose of $200 \mathrm{mg} / \mathrm{kg}$ body weight; Diabetic group received a single IV injection of STZ $(45 \mathrm{mg} / \mathrm{kg})$ body weight; Diabetic+TRF received a single IV injection of STZ $(45 \mathrm{mg} / \mathrm{kg})$ and supplemented with TRF at dose of $200 \mathrm{mg} / \mathrm{kg}$. *: significantly different from the normal group.

Effects of TRF Supplementation on Plasma Levels of TC, TG and Total Lipids

The concentration of TC was significantly higher in the diabetic group compared to the control group $(p=0.0001)$. TRF treatment induced a significant decrease in the concentration of TC in diabetic rats $(p=0.0001)$, restoring the levels of TC to normal conditions. The concentration of plasma TG was significantly higher in TRF-treated and non-treated diabetic rats compared to the control group $(p=0.0001)$ however, TRF treatment did not significantly affect the concentration of TG in diabetic rats. Plasma total lipids levels were markedly increased in untreated diabetic rats compared to the control group $(p=0.0001)$ and TRF significantly reduced the plasma total lipids levels of diabetic rats $(p=0.0001)$ (Figure 2D, 2E and 2F).

\section{Effects of TRF Supplementation on Erythrocyte Membrane Lipid Composition}

STZ-induced diabetic groups (untreated and TRF-treated diabetic rats) showed significantly increased levels of erythrocyte membrane cholesterol when compared to the control group ( $p=0.001$ and $p=0.01$, respectively). However, TRF did not significantly lower the levels of erythrocyte membrane cholesterol in diabetic rats (Table 3).

The composition of phospholipids (phosphatidylinositol, phosphatidylserine, phosphatidylethanolamine, and phosphatidylcholine in erythrocytes membranes were analyzed in Table 3. Our results show that there were lower levels of phosphatidylcholine and higher levels of phosphatidylethanolaminein the diabetic group compared to the control group. Total phospholipids levels were also significantly decreased in both untreated and TRF-treated diabetic rats compared to TRF-treated normal rats ( $p=0.02$ and 0.04 respectively).

Meanwhile, the composition of the analyzed erythrocytes membranes fatty acids are shown in Table 4.

\section{Discussion}

The findings of this study showed that the plasma levels of TC, LDL-C, VLDL-C and TG were elevated in STZ-induced diabetic rats compared to the control group, while the levels of HDL-C were decreased. These findings were in accordance with previous report.(18) Our previous study showed that a daily oral supplementation of $200 \mathrm{mg} / \mathrm{Kg}$ of TRF of palm oil had the beneficial effect of reducing levels of oxidative stress markers by an inhibition of lipid peroxidation and 
an increase in the levels of antioxidant defense system. These results suggest that TRF supplementation plays an important role in reducing oxidative stress-induced diabetes mellitus.(12)
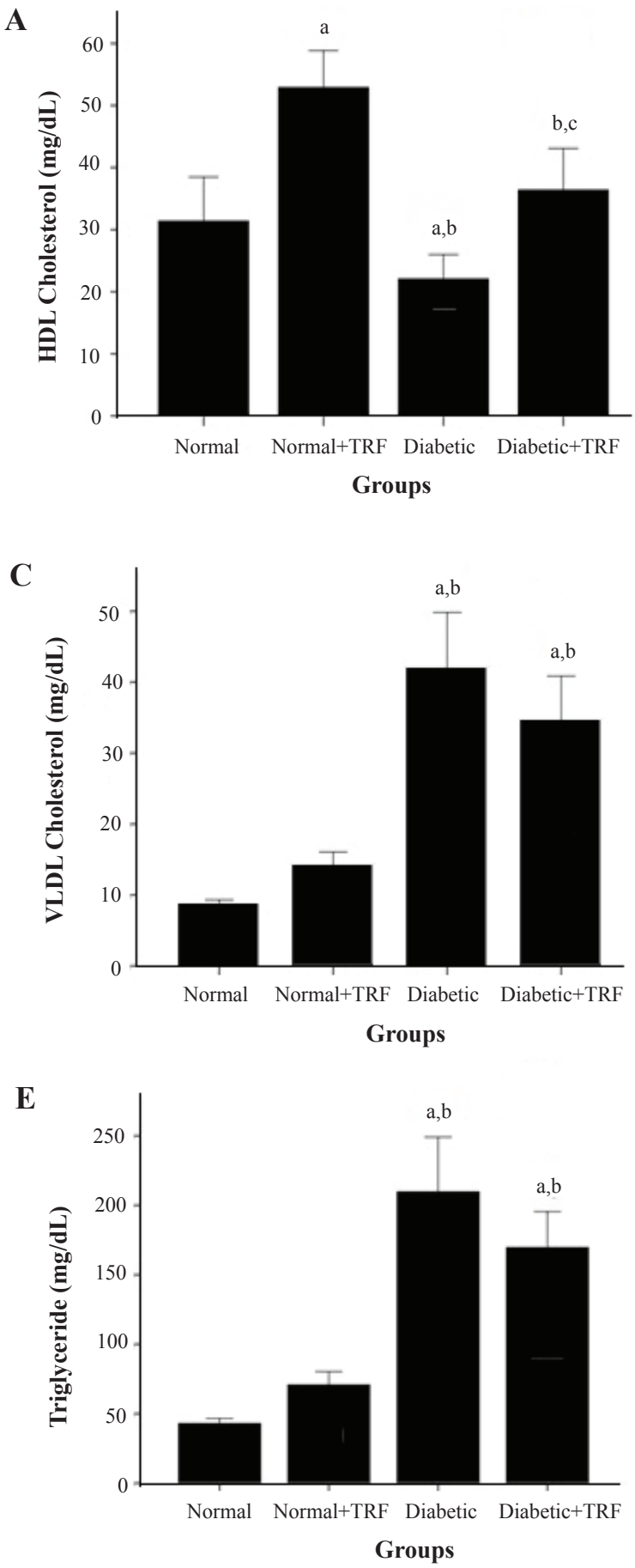

The hypocholesterolemic activity of tocotrienols has recently received much attention, mainly because of their well-established ability to reduce cholesterol biosynthesis. Interestingly, in the present study, the

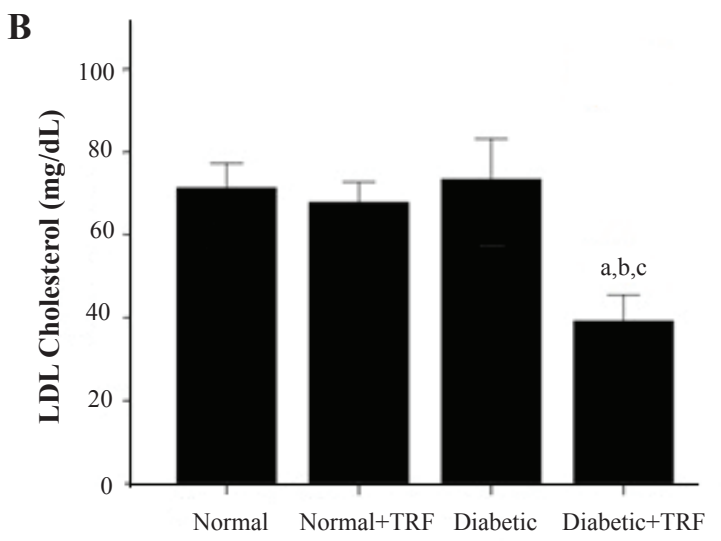

Groups

D

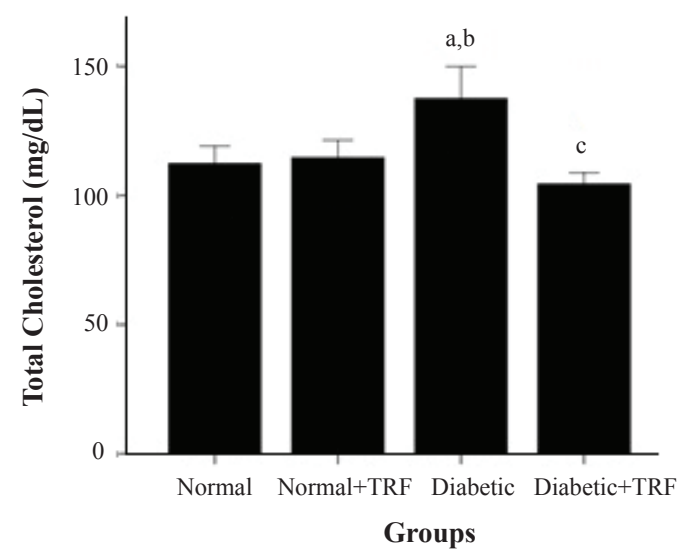

$\mathbf{F}$

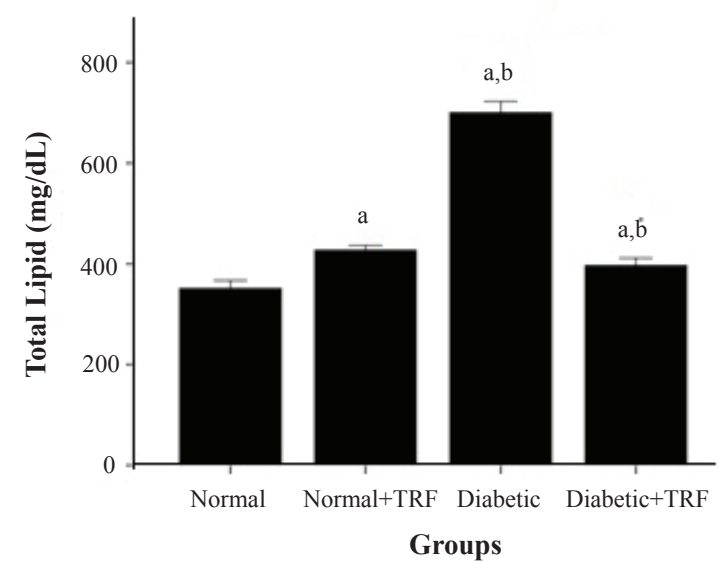

Figure 2. Effects of TRF supplementation on various parameters. A: the effect on HDL-cholesterol; B: the effects on LDL-cholesterol; C: the effect on VLDL-cholesterol; D: the effect on total cholesterol; E: the effects on triglyceride; F: the effects on total lipid. Normal group consist of heatlthy rats; Normal+TRF supplemented with TRF at dose of $200 \mathrm{mg} / \mathrm{kg}$ body weight; Diabetic group received a single IV injection of STZ $(45 \mathrm{mg} / \mathrm{kg}$ ) body weight; Diabetic+TRF received a single IV injection of STZ (45mg/ $/ \mathrm{kg})$ and supplemented with TRF at dose of $200 \mathrm{mg} / \mathrm{kg}$. a: significantly different from the normal group; b: significantly different from the normal+TRF group; c: significantly different from the diabetic group. 
Table 3. Phospholipid compositions of erythrocyte membrane from various experimental groups.

\begin{tabular}{lcccc}
\hline \multicolumn{1}{c}{$\begin{array}{c}\text { Phospholipids Protein } \\
(\boldsymbol{\mu} \mathbf{g} / \mathbf{m g})\end{array}$} & Normal Group & Normal+TRF & Diabetic Group & Diabetic+TRF \\
\hline Phosphatidylcholine & $26.76 \pm 9.30$ & $23.79 \pm 9.00$ & $15.12 \pm 5.60^{\mathrm{a}}$ & $24.44 \pm 11.60$ \\
Phosphatidylinositol & $37.29 \pm 27.10$ & $64.13 \pm 44.20$ & $18.60 \pm 3.80^{\mathrm{b}}$ & $25.32 \pm 13.50^{\mathrm{b}}$ \\
Phosphatidylserine & $14.03 \pm 10.00$ & $24.74 \pm 6.80^{\mathrm{a}}$ & $6.91 \pm 2.90^{\mathrm{b}}$ & $14.20 \pm 8.19^{\mathrm{b}}$ \\
Phosphatidylethanolamine & $86.89 \pm 15.20$ & $76.82 \pm 11.20$ & $91.95 \pm 15.70$ & $78.45 \pm 24.20$ \\
Total phospholipid & $165.00 \pm 42.60$ & $189.40 \pm 57.50$ & $132.60 \pm 12.70^{\mathrm{b}}$ & $142.50 \pm 35.00^{\mathrm{b}}$ \\
Cholesterol & $55.73 \pm 11.50$ & $57.24 \pm 11.30$ & $97.99 \pm 12.00^{\mathrm{a}, \mathrm{b}}$ & $82.73 \pm 19.30^{\mathrm{a}, \mathrm{b}}$ \\
Cholesterol/phospholipid ratio & $0.33 \pm 0.05$ & $0.30 \pm 0.04$ & $0.73 \pm 0.05^{\mathrm{a}, \mathrm{b}}$ & $0.58 \pm 0.08^{\mathrm{a}, \mathrm{b}}$ \\
\hline
\end{tabular}

Results are expressed as means \pm SD. a: significantly different from the normal group at $p<0.05$, b: significantly different from the normal+TRF group at $p<0.05$.

dietary supplementation of TRF (200 $\mathrm{mg}$ for four weeks) significantly reduced the plasma levels of TC and LDL-C and increased the levels of HDL-C in diabetic rats. Increases in plasma HDL-C have been associated with reduced risk for coronary heart disease.(19) These results suggest that TRF is a protective agent for many diseases including atherosclerosis and coronary heart disease. This also confirms the potential of tocotrienols as effective nutritional agents to treat high cholesterol.

In the present study, increased levels of TG were observed in the untreated diabetic group compared to the control group. However, dietary supplementation of TRF in diabetic rats had no effect on the levels of TG, as well as VLDL-C. It is possible that TRF supplementation for 4 weeks at a dose of $200 \mathrm{mg} / \mathrm{kg}$ is not enough to restore the levels of VLDL-C and TG at a normal level. Furthermore, different tocotrienol subtypes possess various degrees of hypolipidemia activity. The results of this study are consistent with previous study.(20)

Several studies aiming at characterizing the lipids composition of erythrocytes membranes have been previously conducted in diabetic humans and animal models. $(21,22)$ In the present study, we observed an alteration of membrane cholesterol, membrane phospholipids, and TC/ total phospholipids ratio in diabetic erythrocytes compared to erythrocytes from the normal group. An increase in membrane cholesterol reflects the increase of the serum cholesterol concentration (23), which was confirmed in the present study. Both, phospholipids and cholesterol, are accountable for the changes of the erythrocyte membrane. (24) They are also responsible for their biconcave shape and basic structural integrity. In this study, membrane fluidity was further evaluated by calculating cholesterol and phospholipid (CH/PL) ratios. The diabetic groups showed higher erythrocyte $\mathrm{CH}$ levels, leading to an increased $\mathrm{CH} / \mathrm{PL}$ ratio and indicating an increase of the membrane rigidity.(25) Surprisingly, we found that TRF treatment did not significantly reduce the concentration of cholesterol in erythrocytes membranes in diabetic rats. One possible explanation for the lack of cholesterol lowering properties of tocotrienols in vivo may be due to poor absorption or rapid clearance from plasma. The controversial effects of tocotrienols were attributed to the presence of $\alpha$-tocopherol, which was shown to attenuate the effect of the cholesterollowering action of tocotrienols in chickens.(26)

Phosphatidylcholine is an important for normal cellular membrane composition and repair. It is also the major delivery form of the essential nutrient choline. One set of roles is an important substrate of synthesis of the neurotransmitter acetylcholine, and having the function of nourishing the brain and improving intelligence.(27)

Our results show that there were lower levels of phosphatidylcholine and higher levels of Phosphatidylethanolamine in the diabetic group compared to the control group. These findings are in agreement with a previous study, which suggested that this phenomenon may be due to a lower rate of conversion of phosphatidylethanolamine into phosphatidylcholine (25), or a down-regulation of choline-phosphatecytidyl transferase, an enzyme regulating phosphatidylcholine synthesis. Increased phosphatidylethanolamine levels in diabetics have been observed in all cell types studied, while sphingomyelin and phosphatidylcholine levels were decreased in platelets.(28)

Phosphatidylinositol is an integral part of cellular and sub-cellular membranes. It exerts its effect through membrane-mediated events.(29) It also modulates the activity of several important membrane-bound enzymes 
Table 4. Fatty acid compositions of erythrocyte membrane from various experimental groups.

\begin{tabular}{lcccc}
\hline Fatty Acids (weight \%) & Normal Group & Normal+TRF & Diabetic Group & Diabetic+TRF \\
\hline Saturated fatty acids (SFA) & & & & \\
14:00 (Myristic acid) & $1.63 \pm 0.87$ & $1.80 \pm 1.20$ & $2.83 \pm 1.80$ & $1.21 \pm 0.64^{\mathrm{c}}$ \\
16:00 (Palmitic acid) & $8.70 \pm 6.90$ & $8.60 \pm 6.80^{\mathrm{a}}$ & $17.30 \pm 7.10^{\mathrm{a}, \mathrm{b}}$ & $18.4 \pm 5.90^{\mathrm{a}, \mathrm{b}}$ \\
17:00 (Heptadecanoic Acid) & $1.92 \pm 1.60$ & $1.84 \pm 1.40^{\mathrm{c}}$ & $8.58 \pm 6.50^{\mathrm{a}, \mathrm{b}}$ & $5.95 \pm 2.30$ \\
18:00 (Stearic Acid) & $7.82 \pm 2.90$ & $6.16 \pm 2.20$ & $9.81 \pm 3.72$ & $7.08 \pm 1.60$ \\
20:00 (Arachidic Acid) & $2.45 \pm 1.40$ & $4.09 \pm 2.30$ & $3.42 \pm 1.04$ & $1.56 \pm 0.43^{\mathrm{b}}$ \\
22:00 (Docosanoic acid) & $12.60 \pm 5.20$ & $12.07 \pm 5.40$ & $3.67 \pm 0.59^{\mathrm{a}, \mathrm{b}}$ & $5.44 \pm 1.50^{\mathrm{a}, \mathrm{b}}$ \\
Total & $35.10 \pm 6.80$ & $34.50 \pm 6.10$ & $45.60 \pm 17.10^{\mathrm{a}}$ & $39.60 \pm 10.30$ \\
\hline Monounsaturated fatty acid (MUFA) & & & \\
$16: 1$ c9 (Palmitoleic acid) & $1.46 \pm 0.77$ & $2.79 \pm 1.10$ & $2.50 \pm 1.20$ & $3.88 \pm 1.60$ \\
$18: 1$ c9 (Oleic acid) & $8.00 \pm 2.90$ & $8.56 \pm 2.50$ & $1.48 \pm 0.54^{\mathrm{a}, \mathrm{b}}$ & $1.91 \pm 1.00^{\mathrm{a}, \mathrm{b}}$ \\
20:1 c 11 (11-Eicosenoic acid) & $3.04 \pm 1.50$ & $1.13 \pm 0.41^{\mathrm{a}}$ & $1.76 \pm 0.53$ & $2.58 \pm 1.10$ \\
Total & $12.51 \pm 3.80$ & $12.50 \pm 3.70$ & $5.74 \pm 1.5^{\mathrm{a}, \mathrm{b}}$ & $8.38 \pm 4.20$ \\
\hline Polyunsaturated fatty acid (PUFA) & & & & \\
$18: 2$ (Linoleic acid) & $17.77 \pm 6.20$ & $17.46 \pm 6.90$ & $5.95 \pm 2.50^{\mathrm{a}, \mathrm{b}}$ & $9.27 \pm 3.40^{\mathrm{a}, \mathrm{b}, \mathrm{c}}$ \\
18:3 (Alpha-linolenic acid) & $1.64 \pm 0.61$ & $0.92 \pm 0.35^{\mathrm{a}}$ & $0.80 \pm 0.51^{\mathrm{a}}$ & $1.05 \pm 0.53$ \\
20:2 (Eicosadienoic acid) & $0.64 \pm 0.17$ & $0.92 \pm 0.53$ & $1.38 \pm 0.48$ & $2.60 \pm 0.27^{\mathrm{a}, \mathrm{b}, \mathrm{c}}$ \\
Total & $20.06 \pm 6.60$ & $19.30 \pm 7.00$ & $8.14 \pm 2.10^{\mathrm{a}, \mathrm{b}}$ & $13.01 \pm 3.30^{\mathrm{a}}$ \\
\hline
\end{tabular}

Results are expressed as means $\pm \mathrm{SD}$. a: significantly different from the normal group at $p<0.05$, b: significantly different from the normal+TRF group at $p<0.05$; c: significantly different from the diabetic group at $p<0.05$

(e.g., $\mathrm{Na}^{+}-\mathrm{K}^{+}$ATPase, alkaline phosphatase, acetyl-CoA carboxylase and tyrosine hydroxylase) as well as having a role in the insulin signal transduction pathway.(30) It is now clear that phosphatidylinositol affects lipoprotein metabolism both by controlling interfacial interactions and uniquely regulating intracellular signaling pathways. PI therefore directly acts to block the synthesis and storage of cholesteryl ester in the blood stream.(31) In addition, phosphatidylinositolappeared to stimulate reverse cholesterol transport (RCT) by promoting a 30 -fold increase in the rate of clearance of free cholesterol (FC) from the circulation.(32) Furthermore previous study shows that phosphatidylinositol also stimulates RCT and results in a net elimination of cholesterol from the body.

Furthermore, this study showed that the levels of phosphatidylinositol were significantly reduced in untreated diabetic group compared to control group. It has been reported that phosphatidylinositol are rich in arachidonic acid and serve as a significant source of eicosanoids.(33) Interestingly, our results show changes in arachidonic acid levels in erythrocytes membranes. The changes in the lipids and phospholipids composition of erythrocytes membranes observed in the present study may induce changes in the physico-chemical properties of the erythrocyte membrane as well as its fluidity/rigidity.(34)

This study provides evidence that the erythrocyte membrane of diabetic rats contains an abnormal lipid matrix. The enrichment in cholesterol and decreasing of total phospholipids in erythrocytes membranes of diabetic rats shown in this study, suggests that the trans-bilayer lipid distribution is probably impaired in diabetic subjects. It is also noteworthy that the levels of saturated fatty acids were higher in the erythrocytes of diabetic rats than in the erythrocytes of normal rats.

Fatty acid residues, namely saturated fatty acids (SFA), monounsaturated fatty acid (MUFA), and polyunsaturated fatty acid (PUFA) can affect membrane fluidity and permeability.(35) The present study showed that the erythrocyte levels of total SFA were higher in the untreated diabetic group compared to the control group. This increase most likely results from the hyperglycemia observed in diabetic patients. Interestingly, TRF treatment induced a decrease of the heptadecanoic acid (17:00) and stearic acid (18:00) levels in diabetic rats, which suggests 
that TRF has antilipidemic effects. However, the levels of docosanoic acid (22:00) were significantly lower in the diabetic groups. The changes detected in diabetic erythrocyte membrane composition, particularly in palmitic acid (C16) levels, could help deciphering the biophysical properties of membranes, such as the mechanisms regulating their fluidity.(36) Previous study reported that elevated C16:0 fatty acids were positively linked with LDL-C (37), whose levels were increased in the diabetic group in the present study.

In this study, we showed that both diabetic groups (untreated and TRF-treated diabetic rats) showed significantly lower levels of oleic acid 18:1 c9 compared to the normal group, which might be due to the reduced activity of stearoyl CoA desaturase (SCD), enzyme responsible for MUFA synthesis, in diabetic conditions. PUFA are important fatty acids in membrane fluidity and flexibility. (38) Our study showed that alpha-linolenic (ALA 18:3n-6) and linoleic acid (LA, 18:2n-6) levels were significantly lower in the diabetic group compared to controls, which is consistent with a previous study.(39) Interestingly, we observed that TRF treatment also significantly increased the levels of eicosadienoic acid (20:2) in diabetic rats. The diabetic group showed lower total MUFA and PUFA. This may be due to defects in the metabolism of essential fatty acids (EFA). TRF supplementation was able to increase total PUFA levels and reduce the difference in lipid fatty acid composition between the diabetic and control animals, which is consistent with the reported antioxidative properties of vitamin E.(40)

\section{Conclusion}

Our results indicate that daily oral supplementation of 200 $\mathrm{mg} / \mathrm{kg}$ of TRF for 4 weeks improved dyslipidemia in STZinduced diabetic rats. Moreover, our data demonstrated the protective effects of TRF against peroxidation of erythrocyte membrane lipids, in particular of unsaturated fatty acids, and suggest the protective effects of TRF against oxidative stress damage.

\section{References}

1. Johansen JS, Harris AK, Rychly DJ, Ergul A. Oxidative stress and the use of antioxidants in diabetes: linking basic science to clinical practice. Cardiovasc Diabet. 2005; 4: 5. doi: 10.1186/14752840-4-5.
2. Aprioku JS. Pharmacology of free radicals and the impact of reactive oxygen species on the testis. J Reprod Infertil. 2013; 14: 158-72.

3. Shargorodsky M, Matas Z, Zimlichman R. Effect of long-term treatment with antioxidants (vitamin $\mathrm{C}$, vitamin $\mathrm{E}$, coenzyme Q10 and selenium) on arterial compliance, humoral factors and inflammatory markers in patients with multiple cardiovascular risk factors. Nutr Metab. 2010; 7: 55. doi: 10.1186/1743-7075-7-55.

4. Frank D, Chin C, Schrader GP, Eckert G, Rimbach G. Do tocotrienols have potential as neuroprotective dietary factors? Ageing Res Rev. 2012; 11: 163-80.

5. Wilankara C, Sharmaa D, Checkera R, Khana NM, Patwardhana R, Patilb A, et al. Role of immunoregulatory transcription factors in differential immunomodulatory effects of tocotrienols. Free Radic Biol Med. 2011; 51: 129-43.

6. Ahsan H, Ahad A, Iqbal J, Siddiqui WA. Pharmacological potential of tocotrienols: a review. Nutr Metab. 2014; 11: 52. doi: 10.1186/17437075-11-52.

7. Ito M, Kondo Y, Nakatani A. Naruse A. New model of progressive non-insulin-dependent diabetes mellitus in mice induced by streptozotocin. Biol Pharmacol Bull. 1999; 22: 988-9.

8. Weiss RB. Streptozotocin: A review of its pharmacology, efficacy and toxicity. Canc Treat Rep 1982; 66: 427-38.

9. Smith SB, Prior RL, Mersmann HJ. Interrelationship between insulin and lipid metabolism in normal and alloxan-diabetic cattle. J Nutr. 1983; 113: 1002-15.

10. Ikebukuro KA, Yasushi Y, Yuichiro F, Shimpei S, Yutaka O, Haruki $\mathrm{H}$, et al. Treatment of streptozotocin-induced diabetes mellitus by transplantation of islet cells plus bone marrow cells via portal vein in rats1. Transplantation. 2002; 73: 512-18.

11. Budin SB, Yusof KM, Idris MH, Abd Hamid Z, Mohamed J. Tocotrienol-rich fraction of palm oil reduced pancreatic damage and oxidative stress in streptozotocin-induced diabetic rats. Aust J Basic Appl Sci. 2011; 5: 2367-74.

12. Matough FA, Budin SB, Hamid ZA, Mariati AR, Alwahaibi N, Mohamed J. Tocotrienol-rich fraction from palm oil prevents oxidative damage in diabetic rats. Sultan Qaboos Univ Med J. 2014; 14: e95-e103.

13. Dodge JT. Composition of phospholipids and of phospholipid fatty acids and aldehydes in human red cells. J Lipid Res. 1967; 8: 66775 .

14. Folch J, Stanley S. A simple method for the isolation of total lipids from animal tissues. Biol Chem. 1957; 226: 497-509.

15. Patton GM, Fasulo JM, Robins SJ. Separation of phospholipids and individual molecular species of phospholipids by high-performance liquid chromatography. J Lipid Res. 1982; 23: 190-6.

16. Morrison WR. Preparation of fatty acid methyl esters and dimethylacetals from lipids with boron fluoride-methanol. J Lipid Res. 1964; 5: 600-8.

17. Tynan MB, Nicholls DP, Maguire SM, Steele IC, McMaster C, Moore $\mathrm{R}$, et al. Erythrocyte membrane fatty acid composition as a marker of dietary compliance in hyperlipidaemic subjects. Atherosclerosis. 1995; 117: 245-52

18. Roehrs M, Valentini J, Paniz C, Moro A, Charão M, Bulcão R, et al. The relationships between exogenous and endogenous antioxidants with the lipid profile and oxidative damage in hemodialysis patients. BMC Nephrol. 2011; 12: 59-71.

19. Bitzur R, Cohen H, Kamari Y, Shaish A, Harats D. Triglycerides and HDL cholesterol: stars or second leads in diabetes? Diabetes Care. 2009; 32: S373-S7.

20. Yuen KH, Wong JW, Lim AB, Ng BH, Choy WP. Effect of mixedtocotrienols in hypercholesterolemic subjects. Funct Foods Health Dis. 2011; 3: 106-17. 
21. Pearce BC, Parker RA, Deason ME, Dischino DD, Gillespie E, Qureshi AA, et al. Inhibitors of cholesterol biosynthesis. 2. Hypocholesterolemic and antioxidant activities of benzopyran and tetrahydronaphthalene analogues of the tocotrienols. J Med Chem. 1994; 37: 526-41.

22. Qureshi AA, Burger WC, Peterson DM, Elson CE. The structure of an inhibitor of cholesterol biosynthesis isolated from barley. J Biol Chem. 1986; 261: 10544-50.

23. Shinde S, Suryakar AN, Sontakke AN, More UK. Effect of antioxidant vitamin supplementation on erythrocyte membrane composition in Type I diabetes mellitus in context of oxidative stress. Biomed Res. 2010; 21: 156-60.

24. Aski BS, Devarnavadagi BB, Rudrappa G, Kashinath RT. Influence of anti diabetic therapy on plasma lipid profile and its relation to erythrocyte membrane lipid levels in type 2 diabetic subjects. GJMR. 2012; 2012: 12.

25. Allen JC, Boyd LC, Alston-Mills BP, Fenner GP. Determination of membrane lipid differences in insulin resistant diabetes mellitus type 2 in whites and blacks. Nutrition. 2006; 22: 1096-102.

26. Qureshi AA, Pearce BC, Nor RM, Gapor A, Peterson DM, Elson CE. Dietary alphatocopherol attenuates the impact of gammatocotrienol on hepatic 3-hydroxy-3-methylglutaryl coenzyme A reductase activity in chickens. J Nutr. 1996; 126: 389-94.

27. Little A, Levy R, Chuaqui-Kidd P, Hand D. A double-blind, placebo controlled trial of high-dose lecithin in Alzheimer's disease. J Neurol Neurosurg Psychiatry. 1985; 48: 736-42.

28. Labrouche S, Freyburger G, Gin H, Boisseau MR, Cassagne C. Changes in phospholipid composition of blood cell membranes (erythrocyte, platelet, and polymorphonuclear) in different types of diabetes-clinical and biological correlations. Metabolism. 1996; 45: 57-62.

29. Mueller RB, Pical C. Inositol phospholipid metabolism in Arabidopsis. Characterized and putative isoforms of inositol phospholipid kinase and phosphoinositide-specific phospholipase C. Plant Physiol. 2002; 130: 22-46.

30. Allen HG. Role of Membrane Lipids in Developing Insulin Resistance Diabetes Mellitus Type II in Caucasians and African Americans
[Dissertation]. Raleigh: Faculty of North Carolina State University; 2000

31. Burgess JW, Boucher J, Tracey AM, Neville PR, Stamler C, Zachariah $\mathrm{S}$, et al. Phosphatidylinositol promotes cholesterol transport and excretion. J Lipid Res. 2003; 44: 1355-61.

32. Stamler CJ, Breznan D, Neville TA, Viau FJ, Camlioglu E, Sparks DL. Phosphatidylinositol promotes cholesterol transport in vivo. J Lipid Res. 2000; 41: 1214-21.

33. Needleman P, Turk J, Jakschik BA, Morrison AR, Lefkowith JB Arachidonic acid metabolism. Annu Rev Biochem. 1986; 55: 69102.

34. Reshamwala SM, Patil ND. Biochemical changes in erythrocyte membrane in uncontrolled type 2 diabetes mellitus. Indian $\mathrm{J}$ Biochem Biophys. 2005; 42: 250-3.

35. Marini M, Abruzzo PM, Bolotta A, Veicsteinas A, Ferreri C. Aerobic training affects fatty acid composition of erythrocyte membranes. Lipids Health Dis. 2011; 10: 188-92.

36. Maturu P, Varadacharyulu N. Adaptive changes in fatty acid profile of erythrocyte membrane in relation to plasma and red cell metabolic changes in chronic alcoholic men. Hum Exp Toxicol. 2012; 31: 652-61.

37. Sepulveda JL, Tanhehco YC, Frey M, Guo L, Cropcho LJ, Gibson $\mathrm{KM}$, et al. Variation in human erythrocyte membrane unsaturated Fatty acids: correlation with cardiovascular disease. Arch Pathol Lab Med. 2010; 134: 73-80.

38. Ozkan Y, Yilmaz OK, Ihsan AO, Yasemin E. Effects of triple antioxidant combination (vitamin $\mathrm{E}$, vitamin $\mathrm{C}$ and a-lipoic acid) with insulin on lipid and cholesterol levels and fatty acid composition of brain tissue in experimental diabetic and non-diabetic rats. Cell Biol Int. 2005; 29: 754-60.

39. Bassi A, Avogaro A, Crepaldi C, Zambon S, Marin R, Macdonald I, et $a l$. Short-term diabetic ketosis alters n-6 polyunsaturated fatty acid content in plasma phospholipids. J Clin Endocrinol Metab. 1996; 81: $1650-3$

40. Evstigneeva RP, Volkov I, Chudinova V. Vitamin E as a universal antioxidant and stabilizer of biological membranes. Membr Cell Biol. 1998; 12: 151-72. 